\title{
Targets for PSAM2 integrase-mediated site- specific integration in the Mycobacterium smegmatis chromosome
}

\author{
Asunción Seoane, Jesús Navas and Juan M. García Lobo \\ Author for correspondence: Juan M. García Lobo. Tel: +34 42 201948. Fax: +34 42201945. \\ e-mail: jmglobo@medi.unican.es
}

Departamento de Biología Molecular, Facultad de Medicina, Universidad de Cantabria, Cardenal

Herrera Oria s/n, 39011

Santander, Spain

\begin{abstract}
An improved integrative cassette from plasmid pSAM2 has been constructed containing plasmid int and attP genes but excluding the xis gene, which should result in increased stability by suppression of the excision reaction. This cassette was included in both suicide and thermosensitive plasmids and used for integration in Mycobacterium smegmatis. Suicide plasmids containing this cassette integrated at a single site (attB1) in the $M$. smegmatis chromosome. The sequence of the attB1 site has been determined and was identified as a putative tRNA ${ }^{\text {Pro }}$ gene. Thermosensitive plasmids containing the cassette integrated both at the same attB1 site and at other different sites, often giving rise to simultaneous integration at two sites. A second integration site (attB2) has been sequenced, which was located in the region encoding 165 rRNA of one of the two rrn operons of $M$. smegmatis.
\end{abstract}

Keywords: Mycobacterium smegmatis, plasmid pSAM2, integrase, site-specific recombination

\section{INTRODUCTION}

Genetic manipulation is a key procedure for the study of mycobacterial biology and for the engineering of the genus to produce either new vaccines or other attenuated strains. Homologous recombination in mycobacteria is a process which is not fully understood, and seems to work better in fast-growing than in slow-growing pathogenic species. Its use for genetic analysis of this genus is also hampered by the high rates of illegitimate recombination (McFadden, 1996). Site-specific recombination in mycobacteria has not been well studied either. However, this kind of RecA-independent process is simpler than homologous recombination and does not usually require extensive sequence homology between the recombining partners. A single recombinase is sufficient with the assistance of some accessory proteins that are ubiquitous in bacteria. Site-specific recombination may therefore provide a useful approach to the genetic manipulation of mycobacteria.

Mycobacteriophages such as L5 have integrases similar to $\lambda$-integrase which have been successfully used for sitespecific gene integration in mycobacteria (Lee et al.,

The GenBank accession numbers of the sequences reported in this paper are AF004309 (attR1), AF004310 (attL1), AF004311 (attB1), AF004312 (attR2), AF004313 (attL2), AF004314 (attB2) and AF004315 (attP).
1991). Integrative plasmids from Streptomyces are capable of autonomous replication but they can also integrate into the bacterial chromosome through a sitespecific recombination event (Pernodet et al., 1984). The integrative functions of plasmid pSAM2 have been well characterized (Boccard et al., 1989a; Kuhstoss et al., 1991; Smokvina et al., 1991). A gene that encodes an integrase belonging to the $\lambda$-integrase family has been located in pSAM2 (Boccard et al., 1989b). Two identical $58 \mathrm{bp}$ att sequences have been found in the plasmid (attP) and in the Streptomyces ambofaciens chromosome (attB). The attB site overlaps a putative gene encoding a tRNA ${ }^{\text {Pro }}$, conserved in many actinomyces, including mycobacteria (Mazodier et al., 1990). It is not known whether the complete tRNA gene is needed for integration or whether a part of it could be sufficient. The use of the integrative functions of plasmid pSAM2 has already been exploited for Streptomyces vector construction (Smokvina et al., 1990; Kuhstoss et al., 1991). In addition, the presence of a sequence similar to PSAM2 attB in the chromosome of mycobacteria suggests the use of this plasmid for the construction of mycobacterial integrative vectors (Eiglmeier et al., 1991). Integration mediated by the pSAM2 integrase has been demonstrated in Mycobacterium smegmatis (Martín et al., 1991) and shown to be site specific. Here, with the aim of improving both the stability and frequency of the integration of pSAM2-based plasmids 
into the M. smegmatis chromosome, we have developed new integrative plasmids and have analysed their integration sites in the M. smegmatis chromosome.

\section{METHODS}

Plasmid constructions. A $1.4 \mathrm{~kb}$ MscI-BamHI DNA fragment from the plasmid pTSN39 (Martín et al., 1991) containing the int gene and the attP site of PSAM2 was ligated to the EcoRI (Klenow filled)/BamHI sites of the vector pUC18. The resulting plasmid was called pLAS3. The kanamycin-resistance gene from Tn903 obtained from Pharmacia (Kanblock) was inserted as a PstI fragment to the PstI site of pLAS3. This $5.4 \mathrm{~kb}$ plasmid was called pLAS4 and was used as a suicide integrative vector for $M$. smegmatis. An Int ${ }^{-}$derivative of pLAS4 was obtained by introducing a frameshift mutation into its int gene by filling the BglII site as described by Boccard et al. (1989b). This Int plasmid was called pLAS4b.

A thermosensitive Mycobacterium-Escherichia coli shuttle plasmid, carrying the int-attP integrative cassette from pLAS4, was also constructed. The thermosensitive plasmid pCG63 (Guilhot et al., 1992) was linearized with BamHI (Klenow filled)/SphI and then ligated to a $1.6 \mathrm{~kb} P v u \mathrm{II}-S p h \mathrm{I}$ fragment from pLAS3 containing the pSAM2 integrative cassette. The resulting plasmid was called pLAS633. An Int $^{-}$derivative of pLAS633, pLAS633b, was constructed as described above for pLAS4. A restriction and genetic map of these plasmids is shown in Fig. 1.

Isolation of integration events. Purified DNA $(0.1 \mu \mathrm{g})$ of either pLAS4 or pLAS4b was used to electrotransform competent $M$. smegmatis $\mathrm{mc}^{2} 155$ cells. Transformants growing at $37^{\circ} \mathrm{C}$ on plates containing kanamycin at $50 \mu \mathrm{g} \mathrm{ml}^{-1}$ should carry the plasmid integrated in the chromosome. Integration of the thermosensitive plasmid pLAS633 was done as described by Guilhot et al. (1994) with slight modifications. Briefly, pLAS633 was electrotransformed into competent $M$. smegmatis $\mathrm{mc}^{2} 155$ cells and stable transformants were selected on plates containing kanamycin at $30^{\circ} \mathrm{C}$. Independent trans- formants were analysed for the presence of the plasmid and grown in $5 \mathrm{ml}$ kanamycin-supplemented 7H9 medium for $48 \mathrm{~h}$ at $30^{\circ} \mathrm{C}$. Dilutions were plated on kanamycin-containing plates, which were incubated for $24 \mathrm{~h}$ at $30^{\circ} \mathrm{C}$ and then shifted to $42{ }^{\circ} \mathrm{C}$ until colonies developed. Colonies obtained from these plates should carry pLAS633 integrated in the chromosome.

Isolation of chromosomal DNA and Southern blot hybridization. Total DNA from mycobacteria was purified basically as described by Ausubel et al. (1990). Chromosomal DNA to be used as a sequencing template was further purified in $\mathrm{CsCl}$ gradients. Total DNA was separated in agarose gels after treatment with restriction endonucleases and blotted on nylon membranes. Probes were labelled with digoxigenin and hybridization detected by a luminescent assay using the DIG DNA labelling and detection kit (Boehringer Mannheim).

DNA sequencing. Plasmid and chromosomal DNA sequences were determined with the Taq polymerase based fmol cyclesequencing system (Promega), using the primers indicated in the text. Samples of $1.5 \mathrm{pmol}$ primer end-labelled with $\left[\gamma^{32} \mathrm{P}\right] \mathrm{ATP}$ and polynucleotide kinase were mixed with either $6 \mu \mathrm{g}$ chromosomal DNA or $100 \mathrm{ng}$ plasmid DNA. The thermocycling programme included a denaturing step at $95^{\circ} \mathrm{C}$ for $2 \mathrm{~min}$, and then $30 \mathrm{cycles}$ each at $95^{\circ} \mathrm{C}$ for $30 \mathrm{~s}, 50^{\circ} \mathrm{C}$ for $30 \mathrm{~s}$ and $70^{\circ} \mathrm{C}$ for $1 \mathrm{~min}$. The sequences obtained were compared to those in GenBank using the BLAST server at the National Centre for Biotechnology Information.

\section{RESULTS}

\section{Plasmid integration in the chromosome of $M$. smegmatis}

The suicide plasmid pLAS4 was introduced by electroporation into $M$. smegmatis $\mathrm{mc}^{2} 155$. The number of colonies obtained was around 150 per $\mu$ g plasmid DNA. Since this plasmid was unable to replicate in mycobacteria, we assumed that kanamycin-resistant trans-
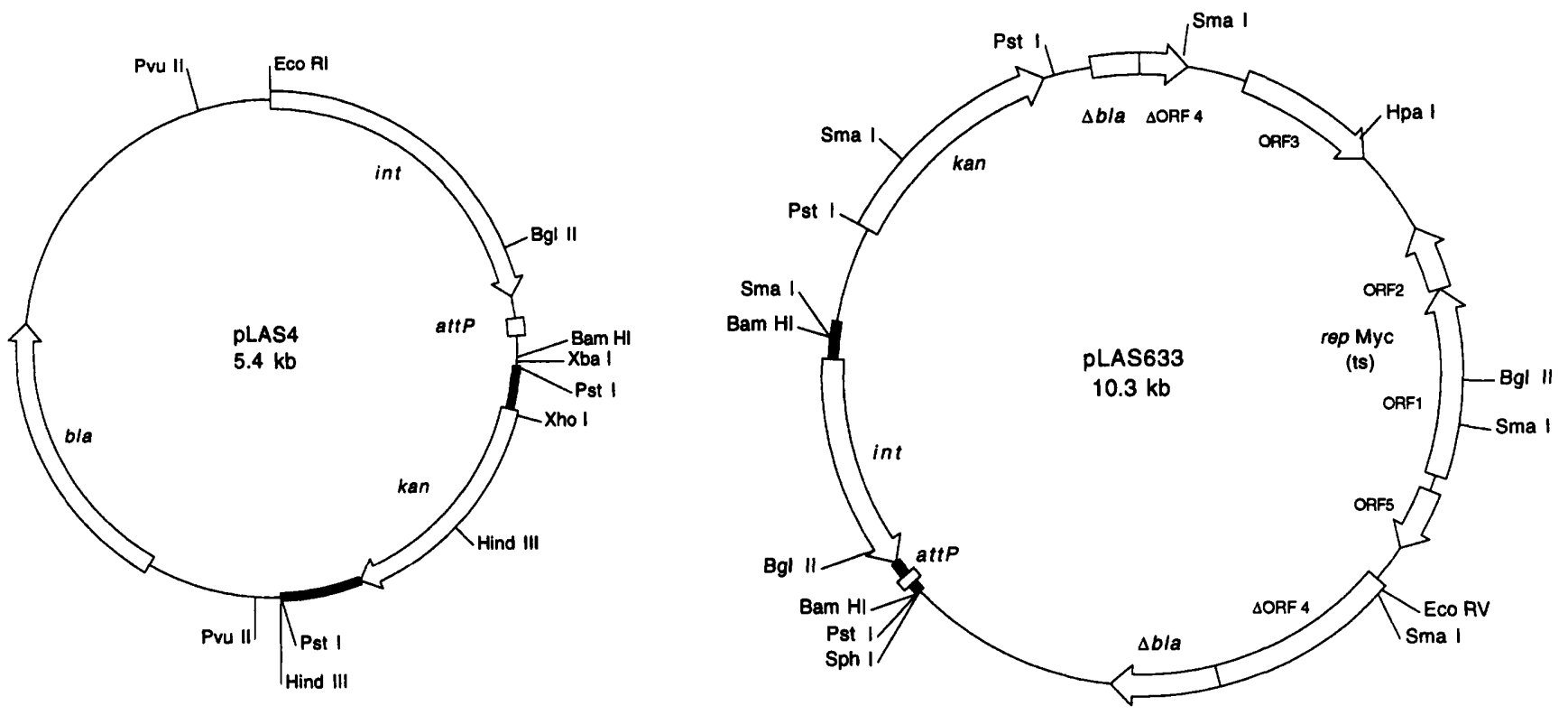

Fig. 1. Restriction and genetic maps of the plasmids pLAS4 and pLAS633. 


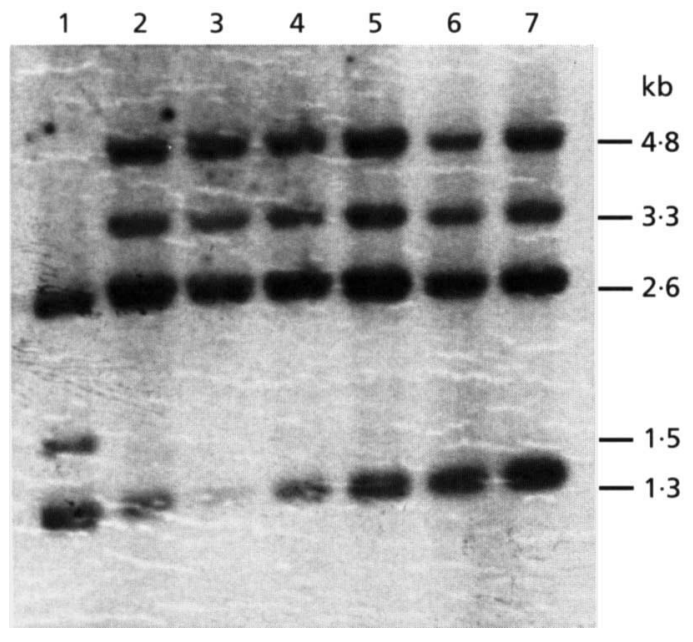

Fig. 2. Hybridization analysis of pLAS4 integration in the $M$. smegmatis chromosome. The complete pLAS4 was labelled and used as a probe. Sizes of the fragments were 2.6, 1.5 and $1.3 \mathrm{~kb}$. (See Fig. 1 for the location of the restriction sites in pLAS4.) The $1.5 \mathrm{~kb}$ fragment contained the attP site. This fragment did not appear in the colonies carrying the plasmid integrated, suggesting that integration occurred through the attP site. The six clones analysed produced the same hybridization pattern (lanes 2-7), indicating that there was a single integration site in the chromosome. Lane 1, plasmid pLAS4 digested with Pvull and $X$ bal.

formants arose as a result of IntpSAM2-mediated integration in the chromosome. This was confirmed by the lack of kanamycin-resistant transformants when the same technique was repeated with the plasmid pLAS4b, an Int $^{-}$derivative of pLAS4.

To improve the integration frequency we performed a similar experiment with the plasmid pLAS633, containing a thermosensitive mycobacterial replicon. The plasmid was introduced into M. smegmatis $\mathrm{mc}^{2} 155$, and kanamycin-resistant transformants were selected at $30^{\circ} \mathrm{C}$, a temperature allowing pLAS633 replication. Colonies able to grow in the presence of kanamycin at $42{ }^{\circ} \mathrm{C}$ were selected as described in Methods. With this procedure we routinely obtained around 2000 kanamycin-resistant colonies per $\mathrm{ml}$ culture. Experiments similar to those described above were performed with plasmid pLAS633b, an Int $^{-}$derivative of pLAS633. In this case, however, no kanamycin-resistant colonies could be obtained after the shift to $42^{\circ} \mathrm{C}$.

\section{Hybridization analysis of the integration sites}

The colonies obtained in the above experiments were checked for the absence of free replicating plasmids by electrophoretic analysis of plasmid lysates and by hybridization of total DNA, with negative results. Total DNA from six colonies obtained from independent experiments that should carry pLAS4 integrated into the chromosome was digested with PvuII and $\mathrm{XbaI}$, blotted and hybridized with pLAS4. The results, shown in Fig. 2 , indicated that the six colonies analysed were identical. $\begin{array}{llllllllll}1 & 2 & 3 & 4 & 5 & 6 & 7 & 8 & 9 & 10\end{array}$

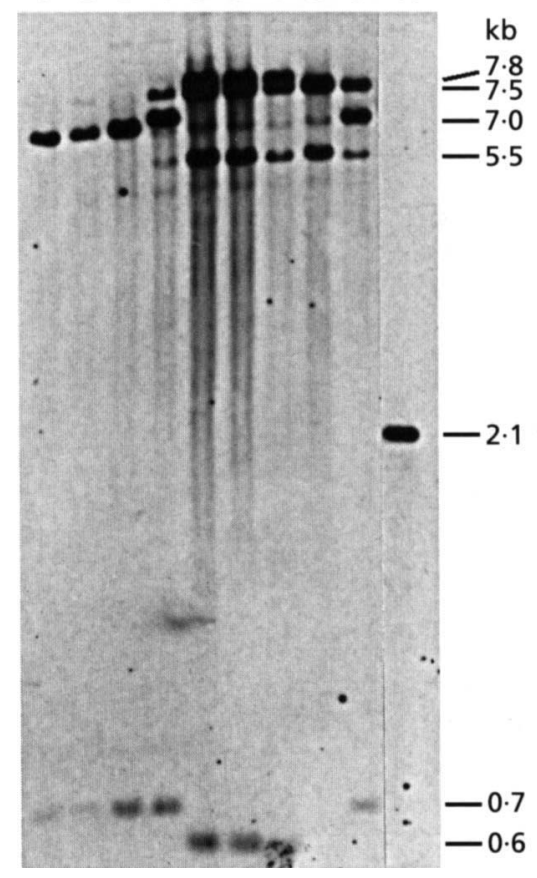

Fig. 3. Hybridization analysis of pLAS633 integration in the $M$. smegmatis chromosome. The probe was a $1.3 \mathrm{~kb}$ fragment containing the PSAM2 int gene and attP site that hybridized with a $2.1 \mathrm{~kb}$ PstI DNA band from pLAS633 (see Fig. 1). This band was absent in all the colonies analysed, demonstrating the absence of free replicating plasmid. Two or four hybridization bands were observed instead, suggesting the presence of one or two integrated copies of the plasmid. Five different hybridization patterns were obtained, corresponding to four different integration sites. The sample in lane 8 shows a single integration at a site that we arbitrarily called site no. 1. Samples in lanes 1,2 and 3 were identical, and contained a single copy of pLAS633 but in a different integration site, site no. 2. Samples in lanes 4 and 9 carry two copies of the plasmid, probably one at site no. 1 and one at site no. 2. Samples in lanes 5, 6 and 7 were identical and they probably carry two plasmid copies, one at site no. 1 and the other at a new site, site no. 3. Lane 10, total DNA from $M$. smegmatis $\mathrm{mc}^{2} 155$ (pLAS633) (unintegrated form) digested with Pstl.

In addition, we found that all colonies contained two of the pLAS4 restriction fragments but the third fragment, containing the attP site, was not present and was replaced by two new fragments formed as a result of the integration event. These results demonstrated that the plasmid was integrated into the chromosome and that integration probably occurred as a result of IntpSAM2mediated site-specific recombination.

In a similar way, total DNA from nine colonies obtained in the integration experiment with pLAS633 was digested with PstI and hybridized against a $1.3 \mathrm{~kb}$ EcoRIBamHI fragment from pLAS3, containing the int-attP region (identical to pLAS633). The probe hybridized to a $2 \cdot 1 \mathrm{~kb}$ PstI fragment of pLAS633. This fragment did not hybridize in any of the recombinant clones, suggesting that the plasmid was indeed integrated into the chromosome. Nevertheless, the nine colonies analysed 


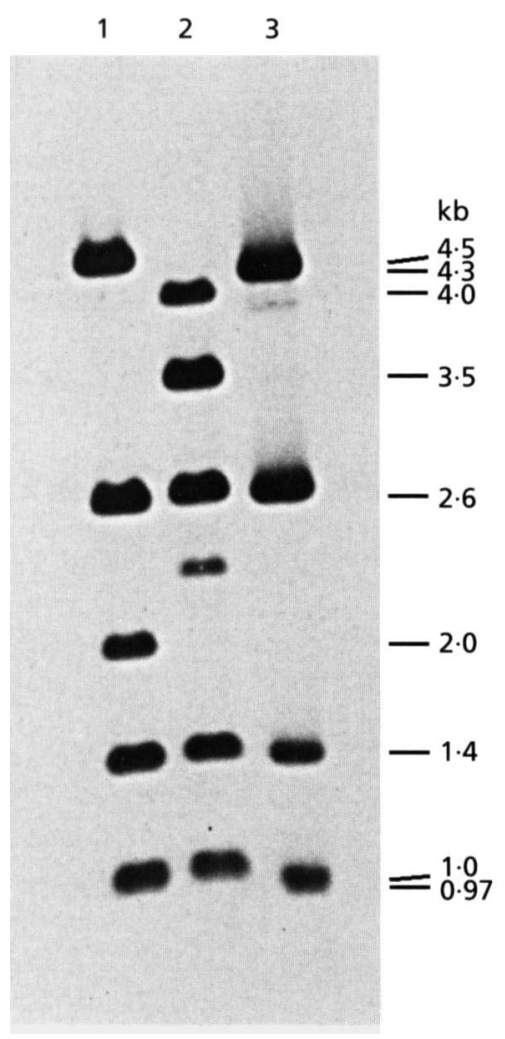

Fig. 4. Southern hybridization analysis of pLAS633 integration with the whole plasmid as a probe to show that the complete plasmid was integrated into the $M$. smegmatis chromosome. DNAs were digested with Smal and hybridized with the whole plasmid pLAS633. Lane 1, single integration at site no. 1; lane 2, single integration at site no. 2; lane 3, purified pLAS633. Plasmid pLAS633 produced five fragments upon digestion with Smal, of $4.3,2.6,1.4,1.0$ and $0.97 \mathrm{~kb}$. The two smaller fragments were not resolved in this gel, and appeared as a double band. The attP site is in the $4.3 \mathrm{~kb}$ fragment. The rep genes are in the 2.6 and the $0.97 \mathrm{~kb}$ fragments (see Fig. 1).

were not identical. At least four different hybridization patterns could be observed, indicating that more than one integration site was being used in the chromosome. In some cases only two hybridization bands were present, suggesting a single integrative event, while in other cases there were four hybridizing bands, suggesting the presence of two copies of the plasmid at different chromosomal positions (Fig. 3).

To show that the complete plasmid was integrated in the chromosome of these colonies, chromosomal DNA from two recombinants was digested with SmaI and probed with pLAS633. The results (Fig. 4) indicated that the whole plasmid was integrated into the chromosome. Analysis of fragment sizes indicated again that the plasmid was probably integrated through the attP site.

\section{DNA sequence of the integration sites}

The nucleotide sequence of pLAS4 around the attP site was verified using as sequencing primer the oligonucleotide TAGTCACGCAGATAGACAC, mapping at the 3'

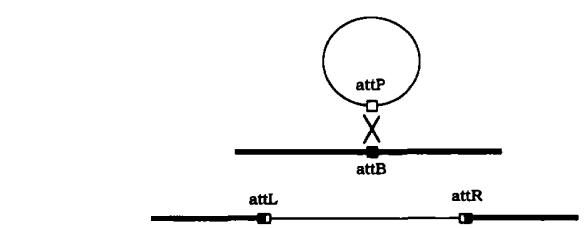

attP

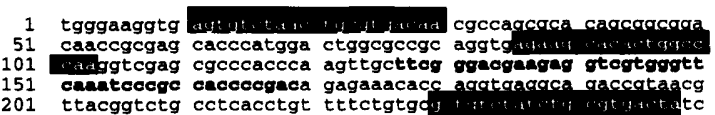

attR1

1 caaggtcgag cgcccaccca agttgcrrco eancanana orcoreoorr 51 CMmMcecec CACCCCancT CGCAGAAGCA AGGCCCTGAC TGGATTCCCA

attL1

1 CAATCTCGGC GCGCGCCTGC GATGTGCCTC TGACCACCCG TTTGGCTTTC 51 AACTGCCTCG GTGCGATACG CTGTCGTGGC TTCACGCGGG GTGTGGCGCA

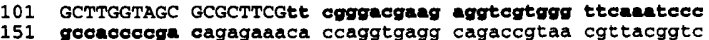
attB1

1 CAATCTCGGC GCGCGCCTGC GATGTGCCTC TGACCACCCG TTTGGCTTTC 51 AACTGCCTCG GTGCGATACG CTGTCGTGGC TTCACGCGGG GTGTGGCGCA

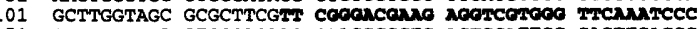
51 accaccccan CTCGCAGAAG CAAGGCCCTG ACTGGATTCC CAGTTCAGGG 201 CCTTTCGCTA TGTGGCCCCA AGTGAGGTAC CGAGCTAC

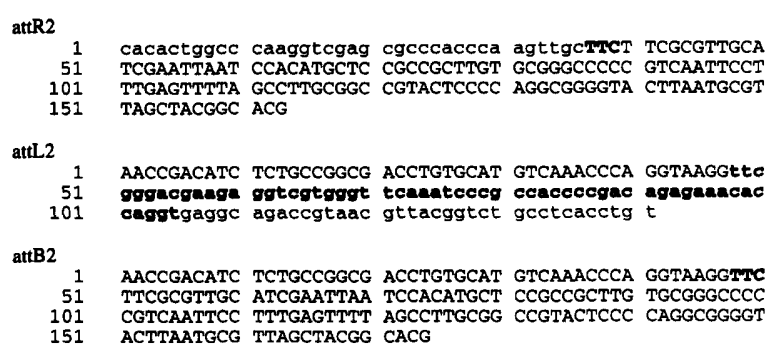

Fig. 5. Nucleotide sequences at the plasmid chromosome junctions of integrations at the attB1 and attB2 sites. The top of the figure shows a model illustrating the recombination event and describing the location of the sequences. The first sequence shown is PSAM2 attP. The sequencing primers are white on a black background. Plasmid sequences are indicated with lower case letters and chromosomal sequences are in upper case. The $43 \mathrm{bp}$ sequence common to attP and attB1 is in bold. The sequences at att $R$ and attL sites and the attB sequences deduced from them are in boxes.

side of attP, according to the sequence reported for pSAM2 attP by Boccard et al. (1989b). The second chain was sequenced with the oligonucleotides AGTGTCTAACTGCGTGACAA and AGAAGCACACTGGCCCAA, deduced from the sequence obtained with the first primer (Fig. 5). The same primers were used to determine the sequence of the att $L$ and att $R$ sequences in the clones carrying the integrated plasmids.

Chromosomal fragments containing the integrated plasmid pLAS4 were cloned taking advantage of the absence of $K p n I$ restriction sites in this plasmid. Chromosomal DNA was digested with $K p n I$, and the fragments were self-ligated and used to transform $E$. coli cells to kanamycin resistance. With this method a $7 \mathrm{~kb}$ plasmid was obtained, and was used to sequence the pLAS4- 
chromosome junctions using the primers described above. The sequences obtained corresponding to the attR1 and attL1 sites are shown in Fig. 5.

The sequence at the attB1 site was deduced from the att $L 1$ and attR1 sequences on the basis of the previously described integration mechanism of pSAM2. The sequence was compared to DNA sequences in GenBank. A region of attB1 was found to be identical to the tRNA ${ }^{\text {Pro }}$ from $S$. ambofaciens and similar to other bacterial tRNA ${ }^{\text {Pro }}$ genes. In addition, the analysis of attB1 with the program tRNAsearch from the PCGENE package revealed that the region between position 87 and position 160 ( 74 bases) of attB1 could be a tRNA ${ }^{\text {Pro }}$ with the anticodon CGG.

In contrast, integration sites of pLAS633 were determined by direct sequencing of chromosomal DNA, due to the lack of restriction enzymes appropriate for the self-ligation approach. Chromosomal DNA was purified from the colonies analysed in Fig. 3. An examination of the sequences revealed that integration in colony 8 had occurred in the previously described attB1 site. However, integration in colonies 1, 2 and 3 occurred at a new site which was identical in all three cases. The attR 2 and at $L 2$ sequences obtained from this site, and the sequence attB2 deduced from them, are shown in Fig. 5. Comparison with sequences in GenBank revealed that the sequence attB2 was identical to a region of the $M$. smegmatis gene encoding the 16S rRNA (Rogall et al., 1990). Sequencing of DNA from colonies 4, 5, 6, 7 and 9 produced mixed sequencing ladders, confirming that they carried at least two plasmid copies in the chromosome and precluding the identification of other integration sites.

\section{Stability of the integrated plasmids}

Loss of the integrated plasmids was investigated through the loss of the kanamycin-resistance marker after growth in a nonselective medium. Three clones carrying each of the integrated plasmids were grown for 56 generations at either $37^{\circ} \mathrm{C}$ (pLAS4) or $42^{\circ} \mathrm{C}$ (pLAS633) and then plated on an antibiotic-free medium. Two hundred colonies from each clone were replicated onto kanamycin plates. All the replicas grew in the presence of kanamycin, indicating that integration was stable, and plasmid loss was lower than $0.5 \%$ after serial passage in a drug-free medium.

\section{DISCUSSION}

In this study we have demonstrated that IntpSAM2mediated site-specific recombination was active in $M$. smegmatis if we introduced a plasmid carrying an integrative cassette containing only the integrase gene and the attP site; attB sequences were present in the $M$. smegmatis chromosome, and any accessory protein necessary for the recombination process was provided by the host.

A different integrative behaviour was observed depending on the type of plasmid used to harbour the integrative cassette. Single insertions at an unique chromosomal location were observed for suicide integrating plasmids. This integration site coincided with the previously described pSAM2 attB. Double insertions involving at least three different integration sites were found when the integrative cassette was placed in a temperaturesensitive plasmid. This difference probably resulted from the time that integrase had to react in the mycobacterial cells. Non-replicating plasmids are expected to be diluted within the population, if not lost, as a result of degradation by the restriction systems of the host. As a result, the level of expression attained by the integrase will be high enough to produce only single, highly specific integrative events at the attB1 site. However, if thermosensitive plasmids carrying the integrative cassette were allowed to grow for some time at the permissive temperature, the integrase had a longer time for expression and to act in the cells, producing multiple integrative events involving more than one integration site. We have sequenced one of the additional sites and found it in one of the two rRNA operons present in the M. smegmatis chromosome. This site was located precisely in the middle of the $16 \mathrm{~S}$ rRNA gene, and was called attB2. Integration at the canonical attB1 site was presumably favoured by the existence of $43 \mathrm{bp}$ of sequence in common between attB1 and attP sites. Although only three common base pairs were observed between the sites attP and attB2, these could be enough to facilitate recombination at the attB2 site as previously demonstrated for integration of the bacteriophage $\phi$ C31(Kuhstoss \& Rao, 1991).

The existence of $43 \mathrm{bp}$ of homology between the attB1 and attP sites prevented determination of the exact location of the crossing-over in the IntpSAM2 recombination reaction at attB1. However, the observation of recombination at attB2, with only $3 \mathrm{bp}$ of sequence homology with attP, allowed us to localize the crossingover site to these $3 \mathrm{bp}$, located in the $5^{\prime}$ end of attP. If is assumed that the same site was used in the recombination at attB1, then the crossing-over site in attB1 could also be precisely located. This position was coincident with the first nucleotide of the anticodon loop of the tRNA as described for other site-specific recombination systems that use tRNA genes as targets. The mycobacteriophage L5 integrates in the chromosome of $M$. smegmatis through a tRNA ${ }^{\text {Gly }}$ gene adjacent to a tRNA ${ }^{\text {Pro }}$ gene (Lee et al., 1991). Interestingly, this gene was slightly different from the tRNA ${ }^{\text {Pro }}$ gene used for pSAM2 integration.

Plasmid integration at the 16S RNA gene of $M$. smegmatis should result in some inactivation of one of the two rRNA operons present in this species (Bercovier et al., 1986). However, no evident differences in growth rate were observed between clones carrying plasmid pLAS633 integrated at attB2 and clones with insertions at attB1.

Integrations of suicide and thermosensitive plasmids were both stable. Excision of pSAM2 has been observed in Streptomyces lividans (Simonet et al., 1987) and S. ambofaciens (Kuhstoss et al., 1989). The increased 
stability of both pLAS4 and pLAS633 integrations in the $M$. smegmatis chromosome can probably be explained by the absence of the PSAM 2 xis gene in these plasmids.

The results reported here represent a new useful tool for the integration of genes into the mycobacterial chromosome and provide further insight into the understanding of the IntpSAM2 mechanism of site-specific recombination.

\section{ACKNOWLEDGEMENTS}

We acknowledge that $\mathrm{A}$. Seoane and J. Navas made equal contributions to this work. We are grateful to C. Martín and B. Gicquel for generously providing plasmid pTSN39 and valuable methodological information, and to Ian Williams for the correction of the manuscript. The work described herein was performed under contract no. BIO2 CT92 0520 with the European Union and cofinanced with grant PB93-0386 from the Spanish DGICYT to J.M.G.L.

\section{REFERENCES}

Ausubel, F. M., Brent, R., Kingston, R. E., Moore, D. D., Seidman, J. G., Smith, J. A. \& Struhl, K. (1990). Current Protocols in Molecular Biology. New York: Wiley.

Bercovier, H., Kafri, O. \& Sela, S. (1986). Mycobacteria possess a surprisingly small number of ribosomal RNA genes in relation to the size of their genome. Biochem Biophys Res Commun 136, 1136-1141.

Boccard, F., Smokvina, T., Pernodet, J. L., Friedmann, A. \& Guerineau, M. (1989a). Structural analysis of loci involved in pSAM2 site-specific integration in Streptomyces. Plasmid 21, 59-70.

Boccard, F., Smokvina, T., Pernodet, J. L., Friedmann, A. \& Guerineau, M. (1989b). The integrated conjugative plasmid pSAM2 of Streptomyces ambofaciens is related to temperate bacteriophages. EMBO J 8, 973-980.

Eiglmeier, K. , Honore, N. \& Cole, S. T. (1991). Towards the integration of foreign DNA into the chromosome of Mycobacterium leprae. Res Microbiol 142, 617-622.

Guilhot, C., Gicquel, B. \& Martín, C. (1992). Temperature-sensitive mutants of the Mycobacterium plasmid pAL5000. FEMS Microbiol Lett 77, 181-186.

Guilhot, C., Otal, I., Van Rompaey, I., Martín, C. \& Gicquel, B. (1994). Efficient transposition in mycobacteria : construction of
Mycobacterium smegmatis insertional mutant libraries. $J$ Bacteriol 176, 535-539.

Kuhstoss, S. \& Rao, R. N. (1991). Analysis of the integration function of the streptomycete bacteriophage $\phi \mathrm{C} 31 . J \mathrm{Mol}$ Biol 222, 897-908.

Kuhstoss, S., Richardson, M. A. \& Rao, R. N. (1989). Site-specific integration in Streptomyces ambofaciens: localization of integration functions in $S$. ambofaciens plasmid pSAM2.J Bacteriol $171,16-23$.

Kuhstoss, S., Richardson, M. A. \& Rao, R. N. (1991). Plasmid cloning vectors that integrate site-specifically in Streptomyces spp. Gene 97, 143-146.

Lee, M. H., Pascopella, L., Jacobs, W. R., Jr \& Hatfull, G. F. (1991). Site-specific integration of mycobacteriophage L5: integrationproficient vectors for Mycobacterium smegmatis, Mycobacterium tuberculosis, and bacille Calmette-Guérin. Proc Natl Acad Sci USA 88, 3111-3115.

McFadden, J. (1996). Recombination in mycobacteria. Mol Microbiol 21, 205-211.

Martín, C., Mazodier, P., Mendiola, M. V., Gicquel, B., Smokvina, T., Thompson, C. J. \& Davies, J. (1991). Site-specific integration of the Streptomyces plasmid pSAM2 in Mycobacterium smegmatis. Mol Microbiol 5, 2499-2502.

Mazodier, P., Thompson, C. \& Boccard, F. (1990). The chromosomal integration site of the streptomyces element pSAM2 overlaps a putative tRNA gene conserved among actinomycetes. Mol Gen Genet 222, 431-434.

Pernodet, J. L., Simonet, J. M. \& Guerineau, M. (1984). Plasmids in different strains of Streptomyces ambofaciens: free and integrated form of plasmid pSAM2. Mol Gen Genet 198, 35-41.

Rogall, T., Wolters, J., Flohr, T. \& Bottger, E. C. (1990). Towards a phylogeny and definition of species at the molecular level within the genus Mycobacterium. Int J Syst Bacteriol 40, 323-330.

Simonet, J. M., Boccard, F., Pernodet, J. L. \& Guerineau, M. (1987). Excision and integration of a self-transmissible replicon of Streptomyces ambofaciens. Gene 59, 137-144.

Smokvina, T., Mazodier, P., Boccard, F., Thompson, C. J. \& Guerineau, M. (1990). Construction of a series of pSAM2-based integrative vectors for use in actinomycetes. Gene 94, 53-59.

Smokvina, T., Boccard, F., Pernodet, J.-L., Friedmann, A. \& Guerineau, M. (1991). Functional analysis of the Streptomyces ambofaciens element pSAM2. Plasmid 25, 40-52.

Received 21 February 1997; revised 13 June 1997; accepted 18 June 1997. 